\title{
Universality and Ambiguities of the Conformal Anomaly
}

\author{
M. Asorey ${ }^{a}$, E. V. Gorbar ${ }^{b, c}$ and I.L. Shapiro ${ }^{b} 1$ \\ (a) Departamento de Física Teorica, Universidad de Zaragoza, 50009, Zaragoza, Spain \\ (b) Departamento de Física - ICE, Universidade Federal de Juiz de Fora, MG, Brazil \\ (c) Bogolyubov Institute for Theoretical Physics, Kiev, Ukraine
}

\begin{abstract}
The one-loop structure of the trace anomaly is investigated using different regularizations and renormalization schemes: dimensional, proper time and Pauli-Villars. The universality of this anomaly is analyzed from a very general perspective. The Euler and Weyl terms of the anomalous trace of the stress tensor are absolutely universal. The pure derivative $\square R$-term is shown to be universal only if the regularization breaks conformal symmetry softly. If the breaking of conformal symmetry by the regularization method is hard the coefficient of this term might become arbitrary which points out the presence of an ambiguous $\int \sqrt{-g} R^{2}$-term in the effective quantum action. These ambiguities arise in some prescriptions of dimensional and Pauli-Villars regularizations. We discuss the implications of these results for anomaly-induced inflationary scenarios and AdS/CFT correspondence.
\end{abstract}

\section{Introduction}

The anomalous violation of local conformal symmetry plays a central role in many applications of quantum field theory in curved space-times (see, e.g., 1 for historic review). The trace anomaly is closely related to the renormalization group and encodes fundamental properties of the Quantum Field Theory in curved spaces which might have cosmological implications. In particular, the modified Starobinsky inflationary model is essentially based on the conformal anomaly (see [2] and references therein). The central idea of this model is the transition from a stable inflation in the UV regime to an unstable one in the IR. This transition is due to the change of sign of the coefficient $a^{\prime}$ of the anomaly-induced $\int \sqrt{-g} R^{2}$-term in the effective action.

The conformal anomaly also played a fundamental role in the verification of the duality relation between string theory and gauge theories unveiled by Maldacena's conjecture [3]. In $\mathcal{N}=4$ SUSY gauge theories the $a^{\prime}$ coefficient of the $\sqrt{-g} R^{2}$ anomalous contribution vanishes if the contribution of each field to $a^{\prime}$ is the standard one. This means that the theory is incompatible with any

\footnotetext{
${ }^{1}$ On leave from Tomsk State Pedagogical University, Russia.
} 
inflationary scenario which is in agreement with the predictions coming from SUGRA in the large $N$ limit [3] and provides a further test of the AdS/CFT correspondence [4].

The conformal anomaly is a well known phenomena arising in the quantization of conformally invariant classical field theories on non-trivial gravitational backgrounds. In four dimensions conformal invariance requires that spinor, vector and scalar fields have to be massless. In scalar field theories conformal invariance also requires that the $\xi$ parameter of the $R \varphi^{2}$ non-minimal interaction must have a fixed value $\xi=1 / 6$.

The trace anomaly is generated by the renormalization of vacuum energy in the presence of an external space-time metric. In the functional integral approach it appears as a consequence of the lack of invariance of the functional measure under conformal transformations. This is reflected in perturbation theory by the appearance of new divergent terms in the effective action which depend on the background space-time metric and vanish for Minkowskian backgrounds. They have to be removed by the corresponding counterterms in order to preserve the renormalizability of the theory (see, e.g. [5] for an introduction). The renormalized effective action which emerges after this procedure is not conformally invariant.

In one-loop approximation the divergent terms are conformally invariant not only for the vacuum energy but also for the matter sector. Conformal invariance is only broken by the divergent coefficients which are of the form $\log M / \mu$ or $\mu^{n-4}$ depending on the regularization method. However, at higher loops, even this marginal conformal invariance may be broken in the scalar field sector, because the $\beta$-function for $\xi$ is not proportional to $\xi-1 / 6$ [6, 7]. Thus, beyond one loop level the theory with $\xi=1 / 6$ is non-renormalizable and new divergent terms of the form $\sqrt{-g} R^{2}$ appear in the vacuum sector. $\int \sqrt{-g} R^{2}$ counterterms have to be included to cancel higher loop divergences according to the prescription of any consistent renormalization scheme and the counterterms are not any more conformally invariant.

In this paper, we shall only focus on one-loop contributions to the conformal anomaly. The main question that we will address is what is the level of universality of the $a^{\prime}$ coefficient of the one-loop $\int \sqrt{-g} R^{2}$ anomalous term of the effective action. The conventional viewpoint establishes that this coefficient $a^{\prime}$ is scheme-dependent because $\sqrt{-g} R^{2}$ is a local term and its coefficient can always be fixed by a renormalization prescription without any restriction. However, most of the renormalization schemes considered in the literature give the same value for $a^{\prime}$, suggesting an unexpected universal behavior. The aim of the paper is to clarify this important issue. For this purpose, we shall consider different regularization schemes, looking for any possible explicit source of ambiguity for this coefficient $a^{\prime}$. The other terms of the one-loop anomaly are linked to non-local terms of the effective action $[8$ and are therefore universal.

The paper is organized as follows. In section 2 a detailed discussion of the anomaly in dimensional regularization is presented. In particular, we show that, contrary to a widespread wisdom [9, 10], the result of the calculation in dimensional regularization does not completely fix the value of the $a^{\prime}$ coefficient. This ambiguity is equivalent to the freedom of adding a $\int \sqrt{-g} R^{2}$-term into the action of the vacuum. In particular, it is possible to define a prescription based on dimensional 
regularization, which provides a value for the $a^{\prime}$ coefficient which is identical to that generated by other regularization schemes like the point-splitting regularization [1] or the quantum effective action method [12. In section 3 we apply the covariant cut-off regularization ${ }^{2}$ of the proper-time integrals to derive the conformal anomaly in the framework of the effective action method [12. In this way we get once more a result consistent with that of Ref. [11. In section 4 we review the derivation of the effective action for the massive scalar field [14] and get identical results that those of [11, 12] when taking the massless limit.

In section 5 we consider a covariant version of the Pauli-Villars regularization. In this regularization the result depends on whether we consider $\xi=1 / 6$ or $\xi \neq 1 / 6$ for the massive Pauli-Villars regulator fields. In the first case the breaking of the conformal symmetry is soft and we get a value $a^{\prime}$ which is consistent with that of [11, 12]. But, if the regulators are not conformally coupled $\xi \neq 1 / 6$, we have a hard breaking of conformal symmetry and the coefficient $a^{\prime}$ becomes arbitrary and is highly dependent on the details of the regularization. This shows that the ambiguity in $a^{\prime}$ first unveiled in dimensional regularization also appears in a regularization scheme with fixed dimensionality $n=4$. Finally, in section 6 we analyze the consequences of this ambiguity. Since the arbitrariness concerns only the initial point of the renormalization group flow there is still room for an inflationary scenario based on the conformal anomaly. However, the results might affect the conjectured AdS/CFT duality unless a similar ambiguity is found in the infrared renormalization of SUGRA in the AdS background.

\section{Dimensional regularization}

To investigate the structure of one-loop vacuum quantum effects, it is sufficient to consider the simplest case: free quantum matter fields on a curved classical background $g_{\mu \nu}$. We shall consider five sorts of quantum fields. The actions of real scalar, Dirac fermion, and massless Abelian vector look as follows (here $d_{i}$ are conformal weights of the fields):

$$
S_{s}=\int d^{4} x \sqrt{-g}\left\{\frac{1}{2} g^{\mu \nu} \partial_{\mu} \varphi \partial_{\nu} \varphi+\frac{\xi}{2} R \varphi^{2}\right\}, \quad d_{s}=-1
$$

where $\xi=1 / 6$ in the four-dimensional, and $\xi=(n-2) / 2(n-1)$ for the $n$-dimensional conformal case,

$$
\begin{array}{ll}
S_{f}=i \int d^{4} x \sqrt{-g} \bar{\psi} \gamma^{\mu} \nabla_{\mu} \psi, & d_{f}=-\frac{3}{2} \\
S_{v}=\int d^{4} x \sqrt{-g}\left\{-\frac{1}{4} F_{\mu \nu} F^{\mu \nu}\right\}, & d_{v}=0 .
\end{array}
$$

In addition, there are two examples of the higher derivative conformal fields: scalar [15]

$$
S_{\mathrm{hds}}=\int d^{4} x \sqrt{-g} \phi\left[\square^{2}+2 R^{\mu \nu} \nabla_{\mu} \nabla_{\nu}-\frac{2}{3} R \square+\frac{1}{3}\left(\nabla_{\mu} R\right) \nabla^{\mu}\right] \phi, \quad d_{\mathrm{hds}}=0
$$

\footnotetext{
${ }^{2}$ Dimensional regularization has been used in Ref. [12] in the way suggested by Brown and Cassidy 13
} 
and spinor [16

$$
S_{\mathrm{hdf}}=i \int d^{4} x \sqrt{-g} \bar{\chi} \gamma^{\mu}\left[\nabla_{\mu} \square+R_{\mu \rho} \nabla^{\rho}-\frac{5}{12} R \nabla_{\mu}-\frac{1}{12}\left(\nabla_{\mu} R\right)\right] \chi, \quad d_{h d f}=-\frac{1}{2} .
$$

The functional integral over all five types of the fields leads to different coefficients in the conformal anomaly of the trace of the dynamical Energy-Momentum (stress) tensor, which is defined by

$$
T^{\mu \nu}=-\frac{2}{\sqrt{-g}} \frac{\delta S}{\delta g_{\mu \nu}} .
$$

Here $S$ is the classical action of the matter fields (15) with an additional metric dependent term $S_{\text {vac }}=S_{\text {vac }}\left[g_{\mu \nu}\right]$ which is necessary for the renormalizability of the theory [10, [5]. Since, quantum corrections of matter fields to the vacuum energy induce divergent terms which depend on the metric, it is necessary to consider a classical action for the metric field in order to absorb the UV divergences by renormalization of the corresponding couplings. In this way we not only renormalize the vacuum energy but also the stress tensor operator which is a composite operator.

The vacuum action $S_{\text {vac }}$ can be regarded as the action of an external metric field, which has not been quantized. From this dynamical viewpoint it might include non-conformal or even non-local terms. However, since we are interested only in quantization of matter fields one has to consider only the terms in $S_{\text {vac }}$ which are absolutely necessary to keep the matter theory renormalizable. This means that in our case $S_{\text {vac }}$ is made of only local terms because the non-local terms are not renormalized. In conformally invariant theories the classical action associated to one-loop counterterms $S_{\text {vac }}$ can be chosen in such a way that it is conformally invariant up to surface terms [5], i.e. it is of the form $S_{\mathrm{vac}}$ (all notations are explained in the Appendix)

$$
S_{\mathrm{vac}}=\int d^{4} x \sqrt{-g}\left\{a_{1} C^{2}+a_{2} E+a_{3} \square R\right\},
$$

where the last term is a pure surface term. With this choice of $S_{\text {vac }}$, the classical conformal Noether identity is satisfied and hence the trace of (6) is zero $T_{\mu}^{\mu}=0$.

In dimensional regularization the counterterms have the form similar to (77) (see, e.g. [10, 5] )

$$
\Delta S_{\mathrm{vac}}=-\frac{\mu^{n-4}}{n-4} \int d^{n} x \sqrt{-g}\left\{\beta_{1} C^{2}+\beta_{2} E+\beta_{3} \square R\right\} .
$$

Here the coefficients $\beta_{i}$ are the $\beta$-functions of the parameters $a_{i}$ of the classical action (7), and $\mu$ is the dimensional parameter of renormalization.

The trace anomaly has the form

$$
T=<T_{\mu}^{\mu}>=-\frac{2}{\sqrt{-g}} g_{\mu \nu} \frac{\delta \Gamma_{\mathrm{vac}}}{\delta g_{\mu \nu}} \neq 0 .
$$

Since the first calculation of the anomaly [9], $T$ has been computed in many different ways (see, e.g. [10, 1] for a list of references, and also [3] for the calculation based on the $A d S / C F T$ correspondence). All methods give an anomaly of the form

$$
T=a C^{2}+c E+a^{\prime} \square R
$$


with $a=\beta_{1}$ and $c=\beta_{2}{ }^{3}$. However, there is a disagreement in the last coefficient $a^{\prime}$.

The conventional result of dimensional regularization [9] is $a^{\prime}=2 \beta_{1} / 3$ while most of the other calculations agree that $a^{\prime}=\beta_{3}$ (such that the anomaly is proportional to the trace of the coincidence limit of the $a_{2}\left(x, x^{\prime}\right)$ coefficient of the Schwinger-DeWitt expansion). Notice that the dimensional regularization also predicts the value $a^{\prime}=\beta_{3}$, but only in the case of global conformal (scale) transformations associated to the renormalization group [17, 15] (see also the discussion in [1, where this was mentioned as an uneasy point). In general, when all five types of the fields are present with different degeneracies $N_{s}, N_{f}, N_{v}, N_{\text {hds }}, N_{\text {hdf }}$, the expressions for the $\beta$-functions are the following

$$
\begin{aligned}
& \beta_{1}=\left(\frac{1}{120} N_{s}+\frac{1}{20} N_{f}+\frac{1}{10} N_{v}\right)-\left(\frac{1}{15} N_{\text {hds }}+\frac{1}{60} N_{\text {hdf }}\right) \\
& \beta_{2}=-\left(\frac{1}{360} N_{s}+\frac{11}{360} N_{f}+\frac{31}{180} N_{v}\right)+\left(\frac{7}{90} N_{\text {hds }}+\frac{3}{40} N_{\text {hdf }}\right) \\
& \beta_{3}=\frac{1}{180} N_{s}+\frac{1}{30} N_{f}-\frac{1}{10} N_{v}-\frac{2}{45} N_{\text {hds }}-\frac{68}{45} N_{\text {hdf }} .
\end{aligned}
$$

It is easy to see that for the ordinary spinors $N_{f}$ and scalars $N_{s}$ the expressions $a^{\prime}=2 \beta_{1} / 3$ and $a^{\prime}=\beta_{3}$ do coincide, while for the three other fields the contribution provided by dimensional regularization differs from that arising in other regularization schemes. Because of this fact we shall consider the coincidence of $N_{f}$ and $N_{s}$ contributions as an accidental fact. We shall try to better understand the origin of the difference between dimensional and other regularizations at one-loop level.

In $\mathcal{N}=4$ supersymmetric $S U(N)$ gauge theories $N_{s}=6\left(N^{2}-1\right), \quad N_{f}=2\left(N^{2}-1\right), \quad N_{v}=$ $\left(N^{2}-1\right), N_{\text {hds }}=N_{\text {hdf }}=0$, also $\beta_{1}=-\beta_{2}$ and $a^{\prime}=0$. This is in agreement with the prediction of the AdS/CFT correspondence as was verified by Henningson and Skenderis [3]. However, the cancelation of the coefficient $a^{\prime}$ does not hold in dimensional regularization, because according to [9] $a^{\prime}=2 \beta_{1} / 3 \neq 0$. It will be very interesting to investigate if there is an infrared regularization of SUGRA in 5-dimensions able to predict a different value for this coefficient.

This value of $a^{\prime}$ can be modified by adding a finite term

$$
S_{+}=\alpha \int d^{4} x \sqrt{-g} R^{2}
$$

to the classical action (7). In order to verify this, we perform a conformal parametrization of the metric

$$
g_{\mu \nu}=\bar{g}_{\mu \nu} e^{2 \sigma}
$$

where the fiducial metric $\bar{g}_{\mu \nu}$ has a fixed determinant. One can establish the following (very useful) identity, which is valid for any functional of the metric $A\left[g_{\mu \nu}\right]$ :

$$
-\frac{2}{\sqrt{-g}} g_{\mu \nu} \frac{\delta A\left[g_{\mu \nu}\right]}{\delta g_{\mu \nu}}=-\left.\frac{1}{\sqrt{-\bar{g}}} e^{-4 \sigma} \frac{\delta A\left[\bar{g}_{\mu \nu} e^{2 \sigma}\right]}{\delta \sigma}\right|_{\bar{g}_{\mu \nu} \rightarrow g_{\mu \nu}, \sigma \rightarrow 0} .
$$

\footnotetext{
${ }^{3}$ In the cosmological setting [2] different notations $w=\beta_{1}, b=\beta_{2}$ and $c=\beta_{3}$ are being used.
} 
Together with the transformation rules derived in the Appendix, this gives

$$
-\frac{2}{\sqrt{-g}} g_{\mu \nu} \frac{\delta}{\delta g_{\mu \nu}} \int d^{4} x \sqrt{-g} R^{2}=12 \square R .
$$

Hence, after including the term (12), the classical trace becomes non-zero $T_{\mu}^{\mu}=12 \alpha \square R$ and the average value acquires exactly the same modification: instead of (10) we have

$$
T=a C^{2}+c E+\left(a^{\prime}+12 \alpha\right) \square R
$$

The finite term (12) of the classical action can be considered as a finite counterterm although strictly speaking, since there are not divergent terms of this type, one might think that it is not necessary to renormalize the theory.

The anomaly can be derived by other regularizations methods. Two important examples are based on point-splitting regularization [11] and the non-local effective action [12, 20]. In both cases one is calculating the anomaly of the Noether identity for the local conformal transformation. Another relevant example is the $\zeta$-regularization 21]. The conventional viewpoint is that the prediction of dimensional regularization [9, 1] disagrees with other regularization methods which give a common result. As it was pointed out in the Introduction, this ambiguity might be very relevant for cosmological applications. Hence it is worth to reconsider the method of [9] and to perform a deeper analysis of the derivation of the anomaly by means of the dimensional regularization. In $n \neq 4$ dimensions the renormalized one-loop action can be split into three parts

$$
\Gamma_{\mathrm{ren}}^{(1)}=S_{\mathrm{vac}}+\bar{\Gamma}^{(1)}+\Delta S_{\mathrm{vac}}
$$

where $\bar{\Gamma}^{(1)}$ is the non-renormalized one-loop quantum correction, which is divergent, conformal invariant and non-local, and $\Delta S_{\text {vac }}$ are the counterterms, which are divergent (to guarantee the finiteness $\Gamma_{\text {ren }}^{(1)}$ ) and local, which remove all UV divergences by renormalizing the parameters $a_{1,2,3}$ of the classical action $S_{\mathrm{vac}}{ }^{4}$. The trace anomaly is generated by the fact that the counterterms $\Delta S_{\mathrm{vac}}$ are not conformal invariant.

The source of arbitrariness come from the fact that the term $C^{2}$ can be extended for $n \neq 4$ in many different ways without violating the minimal requirements of dimensional regularization. The explicit form of $C^{2}(d)$ depends on its dimension $d$ which in general can be of the form $d=$ $n+\gamma(n-4), \gamma$ being an arbitrary real parameter. The integration is $n$-dimensional and the arbitrariness introduced by $\gamma$ is purely algebraic. For any value of $\gamma$ this counterterm is local and covariant, and cancels out the $C^{2}$-type divergence in (16) The choice $\gamma=-1$ has been used in [9], in order to preserve the algebraic form of the counterterm in $\int d^{n} x \sqrt{-g} C^{2}$, although there is no principle imposing such a requirement. With the choice $\gamma=-1$, using Eq. (A17) one gets

$$
T\left(C^{2}\right)=\left.\frac{2}{\sqrt{-g}} g_{\mu \nu} \frac{\delta}{\delta g_{\mu \nu}} \frac{\mu^{n-4}}{n-4} \int d^{n} x \sqrt{-g} \beta_{1} C^{2}(4)\right|_{n \rightarrow 4}=C^{2}-\frac{2}{3} \square R .
$$

\footnotetext{
${ }^{4}$ Alternatively, one can define the vacuum action as $\int d^{4} x \sqrt{-g}\left(\widetilde{a}_{1} R_{\mu \nu \alpha \beta}^{2}+\widetilde{a}_{2} R_{\mu \nu}^{2}+\widetilde{a}_{3} R^{2}\right)$ and renormalize the coefficients $\widetilde{a}_{1}, \widetilde{a}_{2}$ and $\widetilde{a}_{3}$.
} 
Another important observation is that, when the operator (13) is applied to the superficial counterterm $\int d^{n} x \sqrt{-g} \square R$, it gives, automatically, zero. In this way we arrive at the relation $a^{\prime} \equiv-2 \beta_{1} / 3$ advocated in 9 .

On the other hand, the value $\gamma=-1$ is just one of the many possible choices but any other value of $\gamma$ is perfectly valid. Changing the value of $\gamma$ we change the coefficient of the $\square R$-term in (17). Therefore, from this point of view the dimensional regularization incorporates a certain degree of arbitrariness in the determination of the coefficient $a^{\prime}$. This change is equivalent to add the term

$$
\frac{\gamma}{12} \int d^{4} x \sqrt{-g} R^{2}
$$

to the classical action, with $\gamma=12 \alpha$. Thus, the arbitrariness in the choice of $\Delta S_{\mathrm{vac}}$ is equivalent to the addition of a finite counterterm $\int d^{4} x \sqrt{-g} R^{2}$ into the classical action.

One particular value $\gamma=0$ is somehow distinguished, because in this case the counterterm $\int d^{n} x \sqrt{-g} C^{2}(n)$ has, according to Eq. (A16), the simplest conformal transformation law. In fact, with $\gamma=0$ this term transforms exactly as in case of the global conformal transformation $\sigma=$ const. In this case the $\square R$-term of the anomaly is unrelated to the $\int \sqrt{-g} C^{2}$-type counterterm. It is worth to notice that in all other regularization schemes there is no such dependence either. One can argue that this dependence is nothing but an artifact of the dimensional regularization. A remark about the possibility of a similar arbitrariness in the dimensional regularization has been raised by Hawking [21].

\section{A Covariant Proper Time Cut-off Regularization}

The relation between the anomaly and the heat kernel formalism for the effective action has been discussed by Vilkovisky et al in 12,20 . The coefficient of the $\square R$-term in the anomaly agrees with the point-splitting regularization result [1] $a^{\prime}=\beta_{3}$. Indeed, the calculations of [20] have been performed by means of dimensional regularization within the formulation given by Brown and Cassidy [13] which is different from the method used in [9]. It is interesting to recalculate the anomaly by this method [20] but without using dimensional regularization. We shall use instead a covariant proper time cut-off regularization scheme to take care of the ultraviolet divergences. An advantage of this method is that it does not require an extension of the Weyl tensor to the dimensions $n \neq 4$, while allowing to preserve covariance.

This regularization scheme employs the Schwinger proper time formalism [19] and an explicit cut-off on the lower limit of integration over proper time. Practical calculations are very close to the ones of Ref. [20, hence we shall use the same notations. To calculate the $\square R$-term of the conformal anomaly, it is enough to know the effective action up to the second order in curvature. By using the heat kernel calculations of [12] for the operator

$$
\widehat{1} \square+\widehat{P}-\frac{\widehat{1}}{6} R
$$


(here $\widehat{1}$ and $\widehat{P}$ are the identity matrix and the $c$-number operator in the corresponding space) we obtain the following one-loop effective action in the covariant proper time cut-off regularization:

$$
\begin{aligned}
S=\frac{1}{2} \int_{1 / \Lambda^{2}}^{\infty} \frac{d s}{s} \frac{\sqrt{-g}}{(4 \pi s)^{2}} \operatorname{tr} & \left\{\widehat{1}+s \widehat{P}+s^{2}\left[\widehat{1} R_{\mu \nu} f_{1}(-s \square) R^{\mu \nu}+\widehat{1} R f_{2}(-s \square) R\right.\right. \\
+ & \left.\left.\widehat{P} f_{3}(-s \square) R+\widehat{P} f_{4}(-s \square) \widehat{P}+\mathcal{R}_{\mu \nu} f_{5}(-s \square) \mathcal{R}^{\mu \nu}\right]\right\} .
\end{aligned}
$$

$\Lambda$ is a parameter of the covariant cut-off regularization (we shall call it $1 / \Lambda^{2}$-regularization), the functions $f_{i}$ are defined in [12] and $\mathcal{R}_{\mu \nu}$ is defined as a commutator of two covariant derivatives acting on a field $\phi$

$$
\left[\nabla_{\mu} \nabla_{\nu}-\nabla_{\nu} \nabla_{\mu}\right] \phi=\mathcal{R}_{\mu \nu} \phi
$$

In contrast to the conventional dimensional regularization the first two terms in (18) give nonzero contributions to the cosmological constant and the Einstein term, which diverge as $\Lambda^{4}$ and $\Lambda^{2}$, respectively. However, by subtracting the corresponding counterterms, we can completely cancel these contributions. However, the situation is different for terms which are quadratic in curvature. By subtracting the appropriate local counterterms, one gets a finite effective action which contains non-local terms with $\ln \left(-\square / \mu^{2}\right)$, that generate the conformal anomaly. The actual calculation of the anomaly in the $1 / \Lambda^{2}$-regularization is very simple because formulas (4.6)-(4.10) of [12] remain valid and formula (4.11), which defines the conformal variation of action, is replaced by

$$
\delta_{\sigma} S=\frac{1}{2(4 \pi)^{2}} \int d^{4} x \sqrt{-g} \operatorname{tr} \sigma \cdot\left[\square \widetilde{t}_{1}\left(\frac{1}{\Lambda^{2}}, \square\right) \widehat{P}+\square \widetilde{t}_{2}\left(\frac{1}{\Lambda^{2}}, \square\right) R \widehat{1}+O\left(R^{2}\right)\right],
$$

where

$$
\begin{gathered}
\widetilde{t}_{1}(s, \square)=\frac{f(-s \square)-1}{s \square}, \quad \widetilde{t}_{2}(s, \square)=\frac{f(-s \square)-1}{12 s \square}-\frac{f(-s \square)-1-s \square / 6}{2(s \square)^{2}}, \\
f(-s \square)=\int_{0}^{1} d \alpha \exp [\alpha(1-\alpha) s \square] .
\end{gathered}
$$

One can expand $\widetilde{t}_{1}\left(1 / \Lambda^{2}, \square\right)$ and $\widetilde{t}_{2}\left(1 / \Lambda^{2}, \square\right)$ into series in $1 / \Lambda^{2}$. Retaining the leading term, we find

$$
\widetilde{t}_{1}(0, \square)=\frac{1}{6}, \quad \widetilde{t}_{2}(0, \square)=\frac{1}{180}
$$

and, consequently, obtain the same result for anomaly that [12], where the Brown-Cassidy regularization scheme 13] was adopted. In this version of the dimensional regularization scheme there is no room for ambiguity.

In order to illustrate how this scheme works in practice, let us perform the calculations for the particular case of a massless vector, where the conventional dimensional regularization and the method of [13, 12] give different results. In this case the operator has the form

$$
\widehat{H}=H_{\nu}^{\mu}=\delta_{\nu}^{\mu} \square+P_{\nu}^{\mu}-\frac{1}{6} R \delta_{\nu}^{\mu}, \quad \text { where } \quad P_{\nu}^{\mu}=-R_{\nu}^{\mu}+\frac{1}{6} R \delta_{\nu}^{\mu},
$$


and we also have to take into account the contribution to anomaly due to the ghosts

$$
H_{g h}=\square .
$$

A simple calculation shows that $a^{\prime}=\beta_{3}=-1 / 10$, as it was expected from (11).

\section{Anomaly and the massless limit of the massive field theory}

Until now, we have considered only massless fields. However, one can use an alternative approach and try to derive anomaly through a massless limit of massive fields. The importance of this method is related to the fact that quantum corrections coming from massive fields are usually non-local and hence they can not be compensated by adding a local $\int \sqrt{-g} R^{2}$-term into the classical action. Furthermore, from the physical point of view, the concept of massless field is, in many cases, just an approximation valid in the high-energy region.

The derivation of the effective action of massive fields has been performed recently in [14] using the asymptotic expansion for the trace of the heat kernel [22, 12. In 14 the calculations have been performed up to second order in curvature, which is sufficient for our purposes. The universal massless limit has been achieved for the three types of fields (scalars, fermions and vectors), and in all three cases the result is consistent with the point-splitting regularization $a^{\prime}=\beta_{3}$ expression [11]. The result for the massive scalar field with a general non-minimal coupling $\xi$ up to second-order in the curvature part of the effective action is [14]

$$
\begin{gathered}
\bar{\Gamma}_{\text {scalar }}^{(1)}=\frac{1}{2(4 \pi)^{2}} \int d^{4} x g^{1 / 2}\left\{\frac{m^{4}}{2} \cdot\left[\frac{2}{4-n}+\ln \left(\frac{4 \pi \mu^{2}}{m^{2}}\right)+\frac{3}{2}\right]\right. \\
+\left(\xi-\frac{1}{6}\right) m^{2} R\left[\frac{2}{4-n}+\ln \left(\frac{4 \pi \mu^{2}}{m^{2}}\right)+1\right] \\
+\frac{1}{2} C_{\mu \nu \alpha \beta}\left[\frac{1}{30(4-n)}+\frac{1}{60} \ln \left(\frac{4 \pi \mu^{2}}{m^{2}}\right)+k_{W}(a)\right] C^{\mu \nu \alpha \beta} \\
\left.+R\left[\frac{1}{2}\left(\xi-\frac{1}{6}\right)^{2}\left(\frac{2}{4-n}+\ln \left[\frac{4 \pi \mu^{2}}{m^{2}}\right]\right)+k_{R}(a)\right] R\right\} .
\end{gathered}
$$

The form factors are given by

$$
\begin{gathered}
k_{W}(a)=\frac{8 A}{15 a^{4}}+\frac{2}{45 a^{2}}+\frac{1}{150}, \\
k_{R}(a)=A\left(\xi-\frac{1}{6}\right)^{2}-\frac{A}{6}\left(\xi-\frac{1}{6}\right)+\frac{2 A}{3 a^{2}}\left(\xi-\frac{1}{6}\right)+\frac{A}{9 a^{4}} \\
-\frac{A}{18 a^{2}}+\frac{A}{144}++\frac{1}{108 a^{2}}-\frac{7}{2160}+\frac{1}{18}\left(\xi-\frac{1}{6}\right),
\end{gathered}
$$

where

$$
A=1-\frac{1}{a} \ln \frac{1+a / 2}{1-a / 2} \quad \text { and } \quad a^{2}=\frac{4 \nabla^{2}}{\nabla^{2}-4 m^{2}}
$$


If the non-minimal coupling of the theory is conformal $\xi=1 / 6$, the massless limit $m \rightarrow 0$ is non-singular and we arrive at the expected $a^{\prime}=\beta_{3}$ coefficient of the $R^{2}$-term

$$
\bar{\Gamma}^{(1)}(\xi=1 / 6, m \rightarrow 0)=-\frac{1}{12 \cdot 180(4 \pi)^{2}} \int d^{4} x g^{1 / 2} R^{2}+\ldots
$$

Let us remark that in the limit $m \rightarrow \infty$ both form factors $k_{W}(a)$ and $k_{R}(a)$ tend to zero.

\section{Covariant Pauli-Villars regularization}

Pauli-Villars regularization is based on the introduction of a family of massive auxiliary fields which cancel the UV divergences of the theory in a covariant way and formally decouple from the original theory when their masses become very large. The number of the regulator fields, their statistics and masses are chosen in such a way that all the divergences of the theory are canceled. In the singular limit when all their masses simultaneously tend to infinity, only covariant divergences appear and can safely be removed by appropriate renormalization schemes. In the Yang-Mills theories Pauli-Villars regularizations combined with the introduction of higher derivatives regulators in the classical action provided non-perturbative continuum regularization [23, 24], which is very useful to incorporate dynamical fermion fields while keeping all topological properties of gauge fields. In the present context we have only one-loop divergences, and therefore there is no need to apply higher derivatives regularization. For simplicity, we shall restrict our analysis to scalar fields, although the method also works for higher spin fields.

The classical action of the single massless conformal field (10) is replaced by the action

$$
S_{\mathrm{reg}}=\sum_{i=0}^{N} \int d^{4} x \sqrt{-g}\left\{\frac{1}{2} g^{\mu \nu} \partial_{\mu} \varphi_{i} \partial_{\nu} \varphi_{i}+\frac{\xi_{i}}{2} R \varphi_{i}^{2}+\frac{m_{i}^{2}}{2} \varphi_{i}^{2}\right\}
$$

where the physical scalar field $\varphi$ (now labeled by $\varphi_{0}$ ) is conformally coupled $\left(\xi=\frac{1}{6}\right.$ ), massless $\left(m_{0}=0\right)$ and has bosonic statistics $\left(s_{0}=1\right)$. The $N$ Pauli-Villars fields $\varphi_{i} \quad(i=1, \ldots, N)$ are massive $m_{i}=\mu_{i} M \neq 0$ and can have either bosonic $s_{i}=1$ or fermionic statistics $s_{i}=-2$. In the case of having Pauli-Villars fields with identical masses we shall incorporate the multiplicity as a factor in the coefficient $s_{i}$. This means that, in practice, for fields with fermionic statistics $s_{i}$ is a negative even integer whereas for those with bosonic statistics $s_{i}$ may be any natural number. We also assume, for the sake of completeness, that the Pauli-Villars regulators might have non-conformal couplings $\xi_{i} \neq \frac{1}{6}$. The regularized effective action of the massless scalar field with conformal coupling $\xi=\frac{1}{6}$ is given by

$$
\bar{\Gamma}_{\text {reg }}^{(1)}=\lim _{\Lambda \rightarrow \infty} \sum_{i=0}^{N} s_{i} \bar{\Gamma}_{\mathrm{i}}^{(1)}\left(m_{i}, \xi_{i}, \Lambda\right),
$$

where $\Lambda$ is an auxiliar momentum cut-off. 
According to general prescriptions, all divergences in the ultraviolet cut-off $\Lambda$ are canceled out due to the Pauli-Villars conditions

$$
\begin{gathered}
\sum_{i=1}^{N} s_{i}=-s_{0}=-1 ; \\
\sum_{i=1}^{N} s_{i} \mu_{i}^{2}=0 ; \quad \sum_{i=1}^{N} s_{i}\left(\xi_{i}-\frac{1}{6}\right)=0 ; \\
\sum_{i=1}^{N} s_{i} \mu_{i}^{4}=0 ; \quad \sum_{i=1}^{N} s_{i}\left(\xi_{i}-\frac{1}{6}\right)^{2}=0 .
\end{gathered}
$$

The first equation (32 a) cancels out quartic divergences $\Lambda^{4}$, the second and third equations (32. b) cancel quadratic ones $\Lambda^{2}$ and the last two equations (32] c) are required to cancel logarithmic divergences $\log \left(\Lambda^{2} / m^{2}\right)$. A simple solution of these equations matching all these requirements is

$$
\begin{gathered}
s_{1}=1, \quad s_{2}=4, \quad s_{3}=-s_{4}=s_{5}=-2, \\
\mu_{1}^{2}=4, \quad \mu_{2}^{2}=3, \quad \mu_{3}^{2}=1, \quad \mu_{4}^{2}=3, \quad \mu_{5}^{2}=4, \\
\xi_{1}=4+\frac{1}{6}, \quad \xi_{2}=3+\frac{1}{6}, \quad \xi_{3}=1+\frac{1}{6}, \quad \xi_{4}=3+\frac{1}{6}, \quad \xi_{5}=4+\frac{1}{6} .
\end{gathered}
$$

A compact expression of the effective action is obtained in the limit $M \rightarrow \infty$. In this limit the form factors $k_{W}(a)$ and $k_{R}(a)$ for the auxiliary fields vanish and the asymptotics of the remaining expression has the form

$$
\begin{aligned}
\bar{\Gamma}_{\text {reg }}^{(1)}(M)= & \frac{1}{2(4 \pi)^{2}} \int d^{4} x \sqrt{-g}\left\{\frac{M^{4} \alpha}{2}+M^{2} R \beta+\left(\delta-\frac{1}{1080}\right) R^{2}\right. \\
& \left.-\frac{1}{120} C^{\mu \nu \alpha \beta} \ln \left(\frac{\square}{\mathrm{e}^{\gamma} M^{2}}\right) C_{\mu \nu \alpha \beta}\right\}+\mathcal{O}\left(R^{3}, \frac{1}{M}\right)
\end{aligned}
$$

where

$$
\begin{gathered}
\alpha=\sum_{i=1}^{N} s_{i} \mu_{i}^{4} \ln \mu_{i}^{2}, \\
\beta=\sum_{i=1}^{N} s_{i} \mu_{i}^{2}\left(\xi_{i}-\frac{1}{6}\right) \ln \mu_{i}^{2} \\
\gamma=\frac{46}{15}+\sum_{i=1}^{N} s_{i} \ln \mu_{i}^{2} \\
\delta=\sum_{i=1}^{N} s_{i}\left(\xi_{i}-\frac{1}{6}\right)^{2} \ln \mu_{i}^{2}
\end{gathered}
$$

The expression (32) does not include pure divergences like $\square R$ or the Euler term. To obtain these terms would require a more involved calculation specifying the boundary conditions of the fields. The Euler term of the anomaly cannot be inferred from the above expression (32), because it can be derived from higher order terms in the curvature expansion [12] and hence is hidden in the $\mathcal{O}\left(R^{3}\right)$ 
term. Let us remind that here we are using the $\mathcal{O}\left(R^{2}\right)$ approximation described in the previous section.

The Einstein and cosmological constant terms of the regularized action (32) are quartically and quadratically divergent in the large $M$ limit. Since they are local, they can be renormalized in a way which is compatible with conformal invariance. This could be achieved by a suitable choice of the free parameters of the regularization (e.g. $\alpha=\beta=0$ ). The logarithmic divergent term cannot be canceled out by any choice of the parameters of the regularization and need also to be renormalized, but in this case any regularization prescription breaks conformal invariance. Upon renormalization, the regulating massive parameter $M$ is replaced by a renormalization scale $\mu$ which points out the existence of anomaly. The universality of the coefficient multiplying the $\log \mu$ term (replacing $\log M$ ) in the effective action (32), can be understood in very simple terms. It is due to the soldering of this term to that involving the non-local operator $\log \left(\square / \mu^{2}\right)$, which cannot be renormalized and is, therefore, universal [25, 26]. However, the $\int \sqrt{-g} R^{2}$ term has a finite coefficient $\delta-1 / 1080$ which is arbitrary and not linked to any non-local operator. This term, unlike the $\int \sqrt{-g} C^{2}$ term whose coefficient $\gamma / 120$ is also arbitrary, provides a non-trivial contribution to the conformal anomaly unless we choose $\delta=1 / 1080$.

Notice that $\delta$ vanishes if all Pauli-Villars field regulators have conformal couplings $\xi_{i}=\frac{1}{6}, i=$ $1, \ldots N$. This condition is natural since in such a case the Pauli-Villars fields only break conformal invariance through their non-zero masses but do not have an extra breaking due to non-conformal couplings. In this way, we can understand why all other regularizations give a common value for the coefficient $a^{\prime}=\frac{1}{180}$. In most regularizations the breaking of conformal symmetry is soft. However, if we introduce hard breaking regulators like scalar fields with non-conformal couplings we can have an arbitrary contribution $a^{\prime}=\frac{1}{180}-6 \delta$ to the coefficient of $\square R$ term of the trace anomaly. These fields introduce logarithmic divergences in the $\int \sqrt{-g} R^{2}$ term which requires a non-trivial renormalization. The divergences are canceled out in our case by the Pauli-Villars condition (32), but they induce an arbitrary finite contribution which is encoded by $\delta$. A similar phenomenon occurs with any other soft regulator at two loops because in that case the coefficient of the $\int \sqrt{-g} R^{2}$ term becomes divergent as we send the masses of the regulators to infinity. Therefore, it requires a renormalization prescription which fixes its finite value at a given scale. One can notice that dimensional regularization obviously belongs to the same class, because the breaking of conformal symmetry is not soft in this case.

There is no known principle excluding the use of hard regulators or prescribing the exclusive role of soft regulators. Therefore, what we have found is a real ambiguity in the one-loop contribution to the $\int \sqrt{-g} R^{2}$-term. This ambiguity is similar to that found in the renormalization of the ChernSimons coefficient in 3-dimensional gauge theories [25]. In fact, the above interpretation also holds in such a case. The Pauli-Villars regulators which induce the most general effective Chern-Simons coefficient introduce non-trivial logarithmic divergences (i.e. non-trivial beta functions of the gauge coupling constant) which cancel out under Pauli-Villars conditions. But those logarithmic divergent terms can leave a trace in the renormalized value of the Chern-Simons coefficient. This provides for 
the first time a simple explanation in terms of first principles of the striking phenomena observed in Chern-Simons theories 25].

The existence of the ambiguity in the $\int \sqrt{-g} R^{2}$-term does not invalidate the field-theoretical foundations of the anomaly-induced inflation [2]. The beta function of the ambiguous $\int \sqrt{-g} R^{2}$ term in (32) is zero at one loop level, therefore, the actual value of the corresponding coefficient can be fixed by imposing a renormalization condition ${ }^{5}$. Although the quantum corrections to the finite $\sqrt{-g} R^{2}$-term are generally different for distinct regularizations, the renomalized theory may be the same, for the ambiguity can be compensated through the renormalization condition. Despite the one-loop $\beta$-function for the coefficient of the $\sqrt{-g} R^{2}$-term vanishes, the quantum effects induce a universal finite shift in $a^{\prime}$ from the UV to IR. In this sense the flow is universal and independent of the (scheme dependent) initial condition. The universal value of the finite shift for the conformal scalar is $\Delta a^{\prime}=-1 / 1080{ }^{6}$. The existence of this shift is the basis of a mechanism of graceful exit of inflation in the modified Starobinsky scenario when supersymmetric particles of the MSSM model decouple. As a result of the change of the number of active degrees of freedom, the sign of the $a^{\prime}$ coefficient can change from positive in the UV (where the theory is supersymmetric) to the negative in the IR, when supersymmetry is broken. The physical requirements of the change of sign for the $a^{\prime}$ between the UV and IR regimes can be always achieved through the appropriate renormalization condition. One possible solution matching those requirements is to fix $a^{\prime} \approx \beta_{3}$ for all fields [2].

If we assume that the ultimate reason behind the regulators is a more fundamental theory of space-time or string theory the type of regulator should be the same for all particles which survive at lower scales. Therefore, the nature of regulators (hard or soft) is fixed at very high energies and should affect all light particles in the same way. The fact that the choice $a^{\prime}=\beta_{3}$ permits the existence of both inflation and graceful exit mechanisms in a most natural way suggests that the ultimate theory may be associated to soft breaking regulators rather than to hard breaking ones.

In $\mathcal{N}=4$ SUSY gauge theories the existence of ambiguities in the coefficient $a^{\prime}$ implies that there should exist similar ambiguities in the infrared renormalization of supergravity in the bulk if the AdS/CFT correspondence is supposed to be robust under changes of renormalization schemes. Otherwise the correspondence will fail to match the anomaly of the four-dimensional conformal field theory.

\section{Conclusions}

We investigated the universality problem of $\int \sqrt{-g} R^{2}$-terms induced by the conformal anomaly in $4 d$. The results may be summarized as follows:

\footnotetext{
${ }^{5}$ In fact, as it was already mentioned in the Introduction, a renormalization condition for this term is anyway necessary at higher loops because it might become logarithmically divergent.

${ }^{6}$ This flow is very similar to the flow of the central charge in two-dimensional conformal theories where the Zamolodchikov c-theorem 27] establishes a decreasing behaviour of the coefficient of the conformal anomaly from the UV to the IR (see 28] 29] and references therein for extensions to four dimensions).
} 
i) We have verified that the covariant proper-time cut-off renormalization scheme and the massless limit of massive fields lead to identical finite $\int \sqrt{-g} R^{2}$ contributions at one-loop level. This result is perfectly consistent with that of point-splitting regularization method [1] and with the effective action approach of Ref. [12, 20]. This seems to suggest the existence of an unexpected universality of the $\square R$ term in the trace anomaly.

ii) Although it was claimed that the contribution to the coefficient $a^{\prime}$ in dimensional regularization differs from what is obtained in other renormalization schemes [9] we have shown that there is no real disagreement between dimensional regularization and any other regularization scheme. Indeed, the usual way of applying the dimensional regularization [9] can be easily and naturally generalized in such a way that the coefficient of this term and, correspondingly, the coefficient of the $\square R$-term in the trace anomaly become arbitrary.

iii) The ambiguous scheme-dependent $\int \sqrt{-g} R^{2}$-term can also be derived in a covariant Pauli-Villars regularization if the auxiliary massive scalar fields have an additional hard breaking of local conformal symmetry. This ambiguity is scale-independent and therefore can be fixed by the renormalization condition. However, the one-loop renormalization group flows for all three effective charges $a, c, a^{\prime}$ are universal.

iv) Despite the existing ambiguity in the $a^{\prime}$ coefficient, the renormalization group running of the anomaly may be consistent with the requirements of the anomaly-induced inflation scheme. The ambiguity is scale-independent and therefore can be fixed by the renormalization condition.

Finally, it will be very interesting to analize if there are infrared regularizations of SUGRA in 5-dimensions which generate, via the AdS/CFT correspondence, ambiguities similar to those we have found in the conformal anomaly in four dimensions.

\section{Acknowledgments}

We thank I. Avramidi for discussions. M.A. and Ed.G. thank the members of Departamento de Física of UFJF for hospitality. I.Sh. is grateful for the warm hospitality of the Departamento de Física Teórica of the University of Zaragoza, where this work was initiated. The work of M.A. has been partially supported by the Spanish MCyT grant FPA2000-1252. I.S. is indebted to CNPq for the permanent support and to FAPEMIG for the research grant.

\section{Appendix. Conformal transformations in $n$ dimensional space}

In this appendix we summarize the conformal transformation laws of the most relevant geometric invariants in a general $n$-dimensional Riemannian manifold, which have been used in the paper. We use a condensed notations: $A_{\mu}^{2}=g^{\mu \nu} A_{\mu} A_{\nu},(\nabla B)^{2}=g^{\mu \nu} \nabla_{\mu} B \nabla_{\nu} B$ and $\square B=g^{\mu \nu} \nabla_{\mu} \nabla_{\nu} B$. The curvature tensors are defined by

$$
\begin{gathered}
R_{\beta \rho \tau}^{\alpha}=\partial_{\rho} \Gamma_{\beta \tau}^{\alpha}-\partial_{\tau} \Gamma_{\beta \rho}^{\alpha}+\Gamma_{\beta \tau}^{\lambda} \Gamma_{\lambda \rho}^{\alpha}-\Gamma_{\beta \rho}^{\lambda} \Gamma_{\lambda \tau}^{\alpha}, \\
R_{\rho \sigma}=R_{\rho \alpha \sigma}^{\alpha}, \quad R=R_{\alpha}^{\alpha} .
\end{gathered}
$$


Finally, closed parenthesis are used to bound the range of action of derivative operators, e.g. $\nabla_{\mu} B=\left(\nabla_{\mu} B\right)+B \nabla_{\mu}$.

Consider the local conformal transformation

$$
g_{\mu \nu}^{\prime}=g_{\mu \nu} e^{2 \sigma}, \quad \text { where } \quad \sigma=\sigma(x)
$$

The transformation laws for the inverse metric and metric determinant have the form

$$
g^{\prime \mu \nu}=g^{\mu \nu} e^{-2 \sigma}, \quad g^{\prime}=g e^{2 n \sigma}, \quad g=\operatorname{det}\left(g_{\mu \nu}\right) .
$$

For the Christoffel symbols we have

$$
\Gamma_{\alpha \beta}^{\prime \lambda}=\Gamma_{\alpha \beta}^{\lambda}+\delta_{\alpha}^{\lambda}\left(\nabla_{\beta} \sigma\right)+\delta_{\beta}^{\lambda}\left(\nabla_{\alpha} \sigma\right)-g_{\alpha \beta}\left(\nabla^{\lambda} \sigma\right)
$$

and for the curvatures

$$
\begin{aligned}
& R_{\beta \mu \nu}^{\prime \alpha}= R_{\beta \mu \nu}^{\alpha}+\delta_{\nu}^{\alpha}\left(\nabla_{\mu} \nabla_{\beta} \sigma\right)-\delta_{\mu}^{\alpha}\left(\nabla_{\nu} \nabla_{\beta} \sigma\right)+g_{\mu \beta}\left(\nabla_{\nu} \nabla^{\alpha} \sigma\right)-g_{\nu \beta}\left(\nabla_{\mu} \nabla^{\alpha} \sigma\right) \\
&+\delta_{\nu}^{\alpha} g_{\mu \beta}(\nabla \sigma)^{2}-\delta_{\mu}^{\alpha} g_{\nu \beta}(\nabla \sigma)^{2}+\delta_{\mu}^{\alpha}\left(\nabla_{\nu} \sigma\right)\left(\nabla_{\beta} \sigma\right)-\delta_{\nu}^{\alpha}\left(\nabla_{\mu} \sigma\right)\left(\nabla_{\beta} \sigma\right) \\
&+g_{\nu \beta}\left(\nabla_{\mu} \sigma\right)\left(\nabla^{\alpha} \sigma\right)-g_{\mu \beta}\left(\nabla_{\nu} \sigma\right)\left(\nabla^{\alpha} \sigma\right), \\
& R_{\mu \nu}^{\prime}=R_{\mu \nu}-(n-2)\left(\nabla_{\mu} \nabla_{\nu} \sigma\right)-g_{\mu \nu}(\square \sigma)+(n-2)\left(\nabla_{\mu} \sigma\right)\left(\nabla_{\nu} \sigma\right)-(n-2) g_{\mu \nu}(\nabla \sigma)^{2}, \\
& R^{\prime}=e^{-2 \sigma}\left[R-2(n-1)(\square \sigma)-(n-1)(n-2)(\nabla \sigma)^{2}\right] .
\end{aligned}
$$

The Weyl tensor in $n$ dimensions is defined as

$$
\begin{aligned}
C_{\alpha \beta \mu \nu}= & R_{\alpha \beta \mu \nu}+\frac{1}{n-2}\left(g_{\beta \mu} R_{\alpha \nu}-g_{\alpha \mu} R_{\beta \nu}+g_{\alpha \nu} R_{\beta \mu}-g_{\beta \nu} R_{\alpha \mu}\right) \\
& +\frac{1}{(n-1)(n-2)} R\left(g_{\alpha \mu} g_{\beta \nu}-g_{\alpha \nu} g_{\beta \mu}\right) .
\end{aligned}
$$

It is traceless $C_{\mu \alpha \nu}^{\alpha}=0$ and transforms trivially,

$$
C_{\beta \mu \nu}^{\alpha}=C_{\beta \mu \nu}^{\alpha}, \quad \text { i.e. } \quad C_{\alpha \beta \mu \nu}^{\prime}=e^{2 \sigma} C_{\alpha \beta \mu \nu} .
$$

The transformation of the curvature-square scalars are

$$
\begin{aligned}
\sqrt{-g^{\prime}} R_{\mu \nu \alpha \beta}^{\prime 2}= & \sqrt{-g} e^{(n-4) \sigma}\left\{R_{\mu \nu \alpha \beta}^{2}+8 R^{\mu \nu}\left(\nabla_{\mu} \sigma\right)\left(\nabla_{\nu} \sigma\right)-8 R^{\mu \nu}\left(\nabla_{\mu} \nabla_{\nu} \sigma\right)\right. \\
& -4 R(\nabla \sigma)^{2}+4(\square \sigma)^{2}+2(n-2)\left[2\left(\nabla_{\mu} \nabla_{\nu} \sigma\right)^{2}+4(\square \sigma)(\nabla \sigma)^{2}\right. \\
& \left.\left.-4\left(\nabla_{\mu} \nabla_{\nu} \sigma\right)\left(\nabla^{\mu} \sigma\right)\left(\nabla^{\nu} \sigma\right)+(n-1)(\nabla \sigma)^{4}\right]\right\}
\end{aligned}
$$




$$
\begin{aligned}
& \sqrt{-g^{\prime}} R_{\mu \nu}^{\prime 2}= \sqrt{-g} e^{(n-4) \sigma}\left\{R_{\mu \nu}^{2}-2 R(\square \sigma)+(3 n-4)(\square \sigma)^{2}-(n-2)\left[2 R^{\mu \nu}\left(\nabla_{\mu} \nabla_{\nu} \sigma\right)\right.\right. \\
&-2 R^{\mu \nu}\left(\nabla_{\mu} \sigma\right)\left(\nabla_{\nu} \sigma\right)-(n-2)\left(\nabla_{\mu} \nabla_{\nu} \sigma\right)^{2}-(n-1)(n-2)(\nabla \sigma)^{4} \\
&\left.\left.+2 R(\nabla \sigma)^{2}+2(n-2)\left(\nabla_{\mu} \nabla_{\nu} \sigma\right)\left(\nabla^{\mu} \sigma\right)\left(\nabla^{\nu} \sigma\right)-2(2 n-3)(\square \sigma)(\nabla \sigma)^{2}\right]\right\} . \\
& \sqrt{-g^{\prime}} R^{\prime 2}=\sqrt{-g} e^{(n-4) \sigma}\left[R-2(n-1)(\square \sigma)-(n-1)(n-2)(\nabla \sigma)^{2}\right]^{2} .
\end{aligned}
$$

The square of the Weyl tensor

$$
C^{2}(n)=C_{\alpha \beta \mu \nu} C^{\alpha \beta \mu \nu}=R_{\mu \nu \alpha \beta}^{2}-\frac{4}{n-2} R_{\mu \nu}^{2}+\frac{2}{(n-1)(n-2)} R^{2}
$$

transforms as

$$
\sqrt{-g^{\prime}} C_{\mu \nu \alpha \beta}^{2}=\sqrt{-g} e^{(n-4) \sigma} C_{\mu \nu \alpha \beta}^{2} .
$$

One can establish a simple relation between

$$
C^{2}(4)=R_{\mu \nu \alpha \beta}^{2}-2 R_{\alpha \beta}^{2}+1 / 3 R^{2}
$$

and $C^{2}(n)$ :

$$
C^{2}(4)=C^{2}(n)+\frac{2(n-4)}{n-2} R_{\mu \nu}^{2}-\frac{(n-4)(n+1)}{3(n-1)(n-2)} R^{2} .
$$

A similar relation can be derived between $C^{2}(n)$ and $C^{2}(n+\gamma[n-4])$, where $\gamma$ is an arbitrary parameter. Another important combination is

$$
E=R_{\mu \nu \alpha \beta} R^{\mu \nu \alpha \beta}-4 R_{\alpha \beta} R^{\alpha \beta}+R^{2} .
$$

$E$ is the topological density of the Gauss-Bonnet topological term $\int d^{n} \sqrt{-g} E$ in $n=4$. But this term does not contribute to the propagator of gravitons (traceless and completely transverse modes) in any dimension. The conformal transformation of the Gauss-Bonnet term reads

$$
\begin{aligned}
\sqrt{-g^{\prime}} E^{\prime}= & \sqrt{-g} e^{(n-4) \sigma} E+\sqrt{-g} e^{(n-4) \sigma}(n-3)\left[8 R^{\mu \nu}\left(\nabla_{\mu} \nabla_{\nu} \sigma\right)\right. \\
& -8 R^{\mu \nu}\left(\nabla_{\mu} \sigma\right)\left(\nabla_{\nu} \sigma\right)-2(n-4) R(\nabla \sigma)^{2}-4(n-2)\left(\nabla_{\mu} \nabla_{\nu} \sigma\right)^{2} \\
& +4(n-2)(\square \sigma)^{2}+8(n-2)\left(\nabla_{\mu} \sigma\right)\left(\nabla_{\nu} \sigma\right)\left(\nabla^{\mu} \nabla^{\nu} \sigma\right)-4 R(\square \sigma) \\
& \left.+4(n-2)(n-3)(\square \sigma)(\nabla \sigma)^{2}+(n-1)(n-2)(n-4)(\nabla \sigma)^{4}\right] .
\end{aligned}
$$

The last remaining invariant is the surface term with the following transformation rule:

$$
\begin{aligned}
& \sqrt{-g^{\prime}}\left(\square^{\prime} R^{\prime}\right)=\sqrt{-g} e^{(n-4) \sigma}\left[(\square R)-2(n-1)\left(\square^{2} \sigma\right)-(n-1)(n-2) \square(\nabla \sigma)^{2}\right. \\
& +(n-6)\left(\nabla^{\mu} \sigma\right)\left(\nabla_{\mu} R\right)-2 R(\square \sigma)-2(n-1)(n-6)\left(\nabla^{\mu} \sigma\right)\left(\nabla_{\mu} \square \sigma\right) \\
& -(n-1)(n-2)(n-6)\left(\nabla^{\mu} \sigma\right) \nabla_{\mu}(\nabla \sigma)^{2}+4(n-1)(\square \sigma)^{2}-2(n-4) R(\nabla \sigma)^{2} \\
& \left.+2(n-1)(3 n-10)(\square \sigma)(\nabla \sigma)^{2}+2(n-1)(n-2)(n-4)(\nabla \sigma)^{4}\right] .
\end{aligned}
$$




\section{References}

[1] M.J. Duff, Class. Quant. Grav. 11 (1994) 1387.

[2] I.L. Shapiro, Int. J. Mod. Phys. 11D (2002) 1159 hep-ph/0103128;

A.M. Pelinson, I.L. Shapiro and F.I. Takakura, Nucl. Phys. 648B (2003) 417.

[3] M. Henningson and K. Skenderis, JHEP 9807 (1998) 023.

[4] J. Maldacena, Adv. Theor. Math. Phys. 1 (1998) 231.

[5] I.L. Buchbinder, S.D. Odintsov and I.L. Shapiro, Effective Action in Quantum Gravity (IOP Publishing, Bristol, 1992).

[6] L.S. Brown and J.C. Collins, Ann. Phys. 130 (1980) 215.

[7] S.J. Hathrell, Ann. Phys. 139 (1982) 136; 142 (1982) 34.

[8] S. Deser, M.J. Duff and C. Isham, Nucl. Phys. 111B (1976) 45.

[9] M.J. Duff, Nucl. Phys. 125B (1977) 334;

D.M. Capper and M.J. Duff, Nuovo Cim. 23A (1974) 173.

[10] N.D. Birell and P.C.W. Davies, Quantum Fields in Curved Space (Cambridge Univ. Press, Cambridge, 1982).

[11] S.M. Christensen, Phys. Rev. 14D (1976) 2490; 17D (1978) 948; 14D (1976) 2490;

[12] A.O. Barvinsky and G.A. Vilkovisky, Nucl. Phys. 333B (1990) 471.

[13] L.S. Brown and J.P. Cassidy, Phys. Rev. 15D (1977) 1469; 15D (1977) 2810.

[14] E.V. Gorbar and I.L. Shapiro, JHEP 02 (2003) 021; 06 (2003) 006.

[15] R.J. Reigert, Phys. Lett. 134B (1984) 56.

[16] G. de Berredo-Peixoto and I.L. Shapiro, Phys. Lett. 514B (2001) 377.

[17] I.L. Buchbinder, Theor. Math. Phys. 61 (1984) 393.

[18] A.A. Starobinsky, Phys. Lett. 91B (1980) 99.

[19] J. Schwinger, Phys. Rev. 82 (1951) 664.

[20] A.O. Barvinsky, Yu.V. Gusev, G.A. Vilkovisky and V.V. Zhitnikov, Nucl.Phys. 439B (1995) 561.

[21] S.W. Hawking, Comm. Math. Phys. 55 (1977) 133. 
[22] I. G. Avramidi, Yad. Fiz. (Sov. Journ. Nucl. Phys.) 49 (1989) 1185.

[23] A.A. Slavnov, Theor. Math. Phys. 33 (1977) 210;

T.D. Bakeyev and A.A. Slavnov, Mod. Phys. Lett. 11A (1996) 1539.

[24] M. Asorey and F. Falceto, Nucl. Phys. 327B (1989) 427; Phys. Rev. 54D (1996) 5290.

[25] M. Asorey, F. Falceto, J.L. López and G. Luzón, Nucl. Phys. 429B (1994) 344.

[26] J.L. López, Universalidad y Regularización Ultravioleta en Teorías Gauge (Ph.D. dissertation, Zaragoza University, 1995).

[27] A.B. Zamolodchikov, JEPT Lett. 43 (1986) 730.

[28] J.L. Cardy, Phys. Lett. 215B (1988) 749.

[29] S. Forte and J.I. Latorre, Nucl. Phys. 535B (1998) 709. 